\title{
Video Object Segmentation Using Graphs ${ }^{\star}$
}

\author{
Salvador B. López Mármol ${ }^{1,3}$, Nicole M. Artner ${ }^{2}$, Adrian Ion ${ }^{1}$, \\ Walter G. Kropatsch ${ }^{1}$, and Csaba Beleznai ${ }^{2}$ \\ ${ }^{1}$ PRIP, Vienna University of Technology, Austria \\ $\{$ salva, ion, krw\} @prip.tuwien.ac .at \\ 2 Austrian Research Centers GmbH - ARC, Smart Systems Division, Vienna, Austria \\ \{nicole.artner, csaba.beleznai\} @arcs .ac .at \\ 3 Andalusian Research Group FQM-296: "Computational Topology and Applied \\ Mathematics", Spain \\ sallopmar@alum.us.es
}

\begin{abstract}
This paper presents an approach for video object segmentation. The main idea of our approach is to generate a planar, triangulated, and labeled graph that describes the scene, foreground objects and background. With the help of the Kanade-Lucas-Tomasi Tracker, corner points are tracked within a video sequence. Then the movement of the points adaptively generates a planar triangulation. The triangles are labeled as rigid, articulated, and separating depending on the variation of the length of their edges.
\end{abstract}

Keywords: Video object segmentation, adaptive triangulation, articulated objects.

\section{Introduction}

Video object segmentation (VOS) is an important task in computer vision to separate foreground from background and initialize tracking systems. VOS methods can be divided like in [1] into (1) two-frame motion/object segmentation and (2) multi-frame spatio-temporal segmentation/tracking.

Former methods are 23415]. Alatan et al. present in 22 the activities of the COST $211^{\text {ter }}$ group dedicated toward image and video sequence analysis and segmentation, which is an important technological aspect for the success of emerging object-based MPEG-4 and MPEG-7 multimedia applications. In [3], Altunbasak et al. describe a combination of pixel-based and region-based methods to obtain the best possible segmentation results on a variety of image sequences. Castagno et al. present in [4] a scheme for interactive video segmentation. A key feature of the system is the distinction between two levels of segmentation, namely, regions and object segmentation. Chen et al. describe in [5] a method to segment highly articulated video objects with weak-prior random forests. The random forests

\footnotetext{
* Partially supported by the Austrian Science Fund under grants P18716-N13 and S9103-N13.
} 
are used to derive the prior probabilities of the objects's configuration for an input frame. The prior is used to guide the grouping of over-segmented regions.

Latter methods are $16 / 7 / 8$. Celasun et al. write in [6] and [1] about 2-D meshbased VOS. In [8], Tekalp et al. also present a 2-D mesh-based approach. They describe 2-D mesh-based modeling of video objects as a compact representation of motion and shape for interactive, synthetic/natural video manipulation, compression, and indexing. The affine motion model is often used in VOS, because it is simple and locally a good approximation of smooth motion. With only six parameters it is able to describe complex motions like for example rotation, scaling and shearing. Li et al. present in 7] an approach where they use the affine motion model to estimate the motion of homogeneous regions.

Artner et al. present in 9] a kernel-based tracking method using a spatial structure. They show with their experiments that structure (graph-based representation of the target object) can enhance the results of tracking. In [9], the target objects are rigid and the initialization for the tracking process is done manually by selecting the target object.

This paper presents a new approach of VOS, where the result is a triangulated, labeled graph of the scene, which describes foreground objects and background. Each triangle of the graph is labeled either as rigid, articulated or separating, depending on its behavior during a video sequence. We plan to combine the approach in this paper with the method described in [9] to automatically initialize the tracking process and allow to track articulated objects.

The paper is organized as follows: Section 2 recalls the approach in [9]. Section 3 describes our approach. Section 4 shows results with different video sequences. In Section 5 conclusions and future work are given.

\section{Tracking Using Spatial Structure}

Artner et al. propose in [9] an initial concept for combining deterministic tracking of object parts with graph representation encoding structural dependencies between the parts. In general, image graphs can be used to represent structure and topology. The Maximally Stable Extremal Regions (MSER) detector [10] is used in [9] to generate regions which represent the vertices of the graph. The MSER computation is used only once to initialize the graph structure (Delaunay triangulation) and the Mean Shift trackers at each vertex. On the vertices, color histograms are computed to obtain an attributed graph (AG). The edges between the vertices define the region adjacencies.

The objective of Artner et al. is to link the processes of (1) structural energy minimization of the graph and (2) color histogram similarity maximization at the vertices by Mean Shift tracking. The algorithmic combination of Mean Shift and graph relaxation represents a joint iterative mode seeking process on the color similarity and on the structural energy surfaces. As the tracked objects in 9] are rigid, the objective of the relaxation is to maintain the tracked structure as similar as possible to the initial structure. 


\section{Extracting Structure}

Our approach can be divided into 3 steps: track interest points in video with any tracker, build a planar, triangulated graph and analyze the movement of the points (vertices) of the graph over time and label its triangles.

The Kanade-Lucas-Tomasi tracker is used to track corner points. We use the implementation of [11. At the beginning the algorithm selects good features to track and then keeps track of these features. The main idea of the KLT tracker is that feature extraction should not be separated from tracking. If a feature is lost during the tracking process, it is not considered in the following steps of our approach, because it is not dependable.

\subsection{Adaptive Triangulation}

Let $l\left(\mathbf{p}_{\mathbf{1}}, \mathbf{p}_{\mathbf{2}}\right)$ denote the likelihood that two image points $\mathbf{p}_{\mathbf{1}}, \mathbf{p}_{\mathbf{2}}$ belong together i.e. are part of the same (rigid) structure. $l\left(\mathbf{p}_{\mathbf{1}}, \mathbf{p}_{\mathbf{2}}\right)<l\left(\mathbf{p}_{\mathbf{2}}, \mathbf{p}_{\mathbf{3}}\right)$ means that $\mathbf{p}_{\mathbf{1}}, \mathbf{p}_{\mathbf{2}}$ are more likely to be part of the same rigid structure than $\mathbf{p}_{\mathbf{2}}, \mathbf{p}_{\mathbf{3}} . l$ is understood as a nonnegative continuous measure. Note that in the ideal case $l$ should also depend on the type of object considered.

Pairs of points and their properties can be used to decide whether they belong to the same rigid structure or not, but cannot be applied directly to find articulation points. More global information or local information of higher dimensional cells (e.g. triangles) has to be considered. The latter one is used.

A triangulation of a set of points $\mathbf{P} \in \mathbb{R}^{2}$ is a subdivision of the convex hull of the points into triangles. A frequently used triangulation [12] is the Delaunay triangulation [13. The Delaunay triangulation $D T(\mathbf{P})$ of a set of points $\mathbf{P}$ ensures that no point $\mathbf{p} \in \mathbf{P}$ lies within the circumcircle of any triangle formed by other three points. It maximizes the minimum angle of all the angles in the triangulation; it tends to avoid "sliver" triangles. $D T(\mathbf{P})$ is not always unique (e.g. for 4 points on the same circle) and can be computed in $O(n \log n)$ in the number of points. When using $D T(\mathbf{P})$ the presence of edges is decided solely on the position of the points in $\mathbb{R}^{2}$ and additional hints like the likelihood $l$ are hard to integrate. One could imagine finding a new set of points for which the euclidean distance is proportional to $l$ and then compute the triangulation, but such a set does not always exist. The constrained Delaunay triangulation requires a priory knowledge of the edges that are necessarily part of it.

In the ideal case, the used triangulation mainly contains edges with high $l$, increasing the chance that vertices of the same rigid part are connected, and articulation points are correctly detected. The following formulation is used: given a fully connected graph $G=(\mathbf{V}, \mathbf{E})$, where all $\mathbf{v} \in \mathbf{V}$ correspond to a unique point $\mathbf{p} \in \mathbf{P}$ (bijection), and all $\mathbf{e}=\left(\mathbf{v}_{\mathbf{1}}, \mathbf{v}_{\mathbf{2}}\right) \in \mathbf{E}$ are weighted with $l\left(\mathbf{p}_{\mathbf{1}}, \mathbf{p}_{\mathbf{2}}\right)$, where $\mathbf{p}_{\mathbf{1}}, \mathbf{p}_{\mathbf{2}}$ are the points associated to $\mathbf{v}_{\mathbf{1}}$, respectively $\mathbf{v}_{\mathbf{2}}$, find the connected subgraph $T=\left(\mathbf{V}, \mathbf{E}^{\mathbf{T}}\right)$ s.t. $T$ is a triangulation, trying to keep edges with higher likelihoods. Algorithm 1 computes $T$, for a given fully connected graph $G$ with $|\mathbf{V}|>2$. Note that Algorithm 1 converges after maximum $|E|$ steps, as in each iteration at least one edge is removed from $\mathbf{S}$ (Line $\mathbf{7}$ ). 


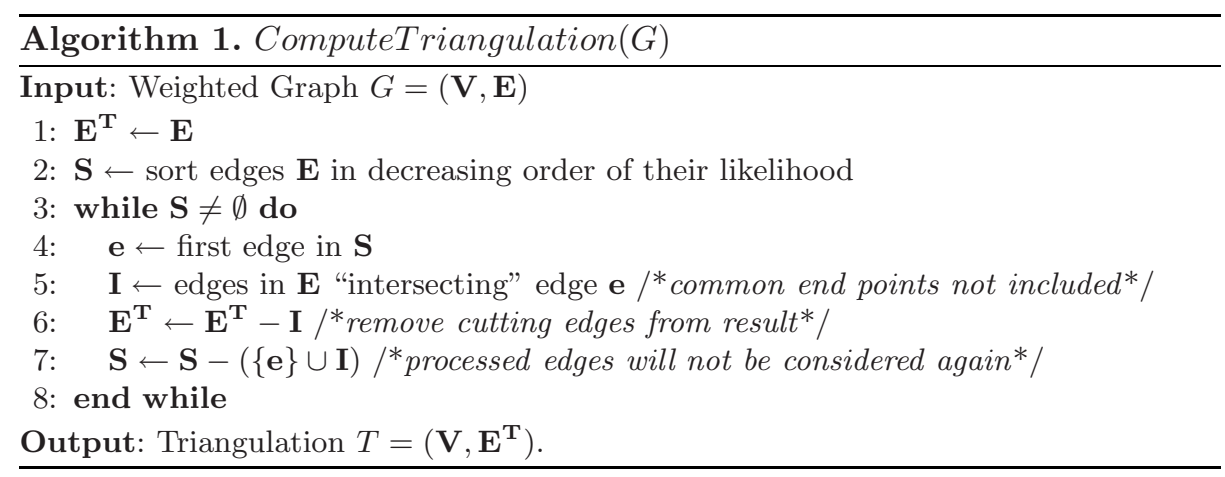

Property 1. If $G$ is a fully connected graph, Algorithm 1 returns a triangulation.

Proof. Assume $T$ contains one face $F$ with $k>3$ vertices. As $G$ is a fully connected graph, all $k$ vertices of the face $F$ are pairwise connected. Any "internal" edge $i$ is removed only if it intersects a "surviving" edge $e$ (Line6 in Algorithm[1). For $F$, the surviving edge $e$ could connect:

- two vertices not part of $F$ : would not produce $F$ as a face, as it would remove also at least 2 other edges of $F$ and disconnect it;

- one vertex of $F$ with one vertex not part of $F$ : would not produce $F$ as a face, as it would remove also at least 1 other edge of $F$ and disconnect it;

- two vertices of $F$, not defining a boundary edge: edge $e$ would divide $F$ in two parts being either triangles or fully connected subgraphs.

Property 2. If $G(\mathbf{V}, \mathbf{E})$ is a supergraph of a triangulation s.t. $\forall e \in \mathbf{E}$, with $\mathbf{I} \subset \mathbf{E}$ all edges intersecting $e, G^{\prime}(\mathbf{V}, \mathbf{E}-\mathbf{I})$ is triangulation or a supergraph of a triangulation with the same property, then Algorithm 1 returns a triangulation. (proof similar to Property 10)

Starting with a fully connected graph is computationally expensive $\left(|E|^{2}=|V|^{4}\right.$ intersection), thus a faster approximation solution is proposed. We propose $G$ to be a "non optimal" triangulation $T_{i}$ to which all end vertices of paths of length two are added. Algorithm 1 is applied to select the triangulation $T$ considering $l$. Note that Property 2 is not satisfied for any triangulation $T_{i}$, and to make sure, additional conditions are necessary (e.g. adding only edges connecting the non common vertices of two adjacent triangles).

We have set $G$ to be $D T(\mathbf{P})$ to which edges connecting all end vertices of paths of length two were added. $l\left(\mathbf{p}_{\mathbf{1}}, \mathbf{p}_{\mathbf{2}}\right)$ was set as detailed in Section 3.2. For $D T(\mathbf{P})$, the points that have been tracked successfully over the whole sequence and their position in the first frame was used. For the previous, Algorithm 1 always produced a triangulation. Figures 2 (a) and 4 (a) show examples of produced triangulations.

An alternative approach to the one in Algorithm 1 would have been to weight the edges with the inverse of the likelihood $l$, build the Minimum Spanning Tree 
of $G$ and add edges with high $l$ to produce a triangulation. Planarity would still have to be ensured, requiring the edge intersections to be done.

\subsection{Motion Analysis and Labeling}

The labeling of the triangles depends on the movement of their vertices $\mathbf{v}$ and so on the variation of the length of their edges $\mathbf{e}$. Every edge $\mathbf{e}=\left(\mathbf{v}_{i}, \mathbf{v}_{j}\right)$ is weighted depending on its variation with $w(\mathbf{e})=l\left(\mathbf{p}_{\mathbf{i}}, \mathbf{p}_{\mathbf{j}}\right)=\max (\mathbf{e})-\min (\mathbf{e})$, where $\max (\mathbf{e})$ and $\min (\mathbf{e})$ are the maximum and the minimum length of edge $\mathbf{e}$ during the whole sequence, respectively. In our experiments, $w(\mathbf{e})$ proved to be robust against repeated small errors in the tracking. The standard deviation was also considered, but constant edge length for a longer time reduces the effect of length variation on the weight.

If $w(\mathbf{e})>\epsilon$, the edge is treated as eventful during the analysis. The threshold $\epsilon$ is used to compensate small variations in the edge length due to discretization, noise or tracking errors. The triangles are labeled with the following rules:

- rigid: The weights of all three edges of a triangle lie over the threshold $\epsilon$.

- articulated: The weight of one edge of a triangle is under $\epsilon$.

- $\boldsymbol{\Delta}$ separating: At least two edges are eventful, which indicates that the triangle is a connection between foreground and background or another object.

In Figure 1, two simple examples of our labeling mechanism are shown. The sequence in the examples consists only of two frames, because of simplicity. In our experiments (see Section 4) we used a full sequence of frames. Figure 1 (a) and (b) show the two frames $E_{a}(1)$ and $E_{a}(2)$ of example $E_{a}$. Due to the movement of the vertices (points) from frame (a) to (b), the square is detected as a rigid foreground object, because its vertices did not move and so its triangles are labeled with rigid (blue). The triangles that connect the square to the background are labeled with $\boldsymbol{\Delta}$ separating (red), which means this edges should be cut to separate the foreground object from the background.

$E_{b}$ in Figure 1 (c) and (d) is a labeling example with all three kinds of labels: - rigid (blue), $\mathbf{\square}$ articulated (green), and $\boldsymbol{\Delta}$ separating (red). One triangle in $E_{b}$ is labeled as articulated, because on edge of the triangle changed its length during the initialization phase over the threshold $\epsilon$.

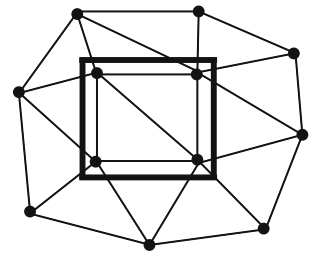

(a) $E_{a}(1)$

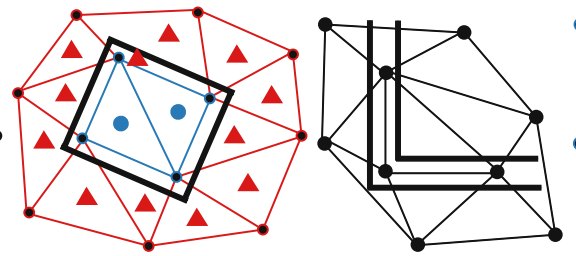

(b) $E_{a}(2)$ (c) $E_{b}(1)$

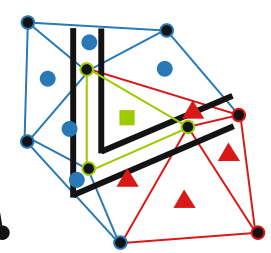

(d) $E_{b}(2)$

Fig. 1. Labeling triangles:

rigid (blue), articulated (green), separating (red) 


\section{Experiments and Discussion}

The KLT Tracker [14 was used to track corner points and the triangulation is build as described in Algorithm 1. Then the movement of the tracked points, grouped in triangles, is analyzed and the graph is labeled (see Section 3.2).

Labeling rigid triangles: In sequence 1 the background and the static objects (left and right) are detected as a rigid structure (Figure 21). The moving candy box (center) is also identified as a rigid object, and the triangles between the candy box and the background are correctly labeled as separating (are not drawn). No articulation points are detected, which is correct too.

Labeling articulated triangles: Video sequence 2 (Figure 3) shows a human moving his head and arms. Articulation points located in shoulder, elbows and neck can be detected. Round stickers were used to provide sufficient corner points for the KLT tracker in structurally interesting positions.

Problems during the tracking process produced some inconsistent results (i.e. some separating and articulation triangles inside the right arm). Nevertheless, the labeling is satisfying as most of the articulation points are located (only the left elbow is not detected) and the rigid and separating triangles are correctly labeled (Figure 4).

Adaptive triangulation vs Delaunay triangulation: For this experiment we used video sequence 2 again. The labeling process is done on the Delaunay triangulation in order to obtain a comparison between Delaunay and the proposed adaptive triangulation. The proposed adaptive triangulation gives higher priority to connections between points keeping the same relative position (e.g. points on left arm and hand in Figure 4). Figure 4 (d) shows how triangles that join objects and background can produce disconnected objects in the labeling on the Delaunay triangulation. The adaptive triangulation manages to keep as many triangles as possible inside the same object, which leads to a better (connected) result after the labeling (see Figure 4 (b)).

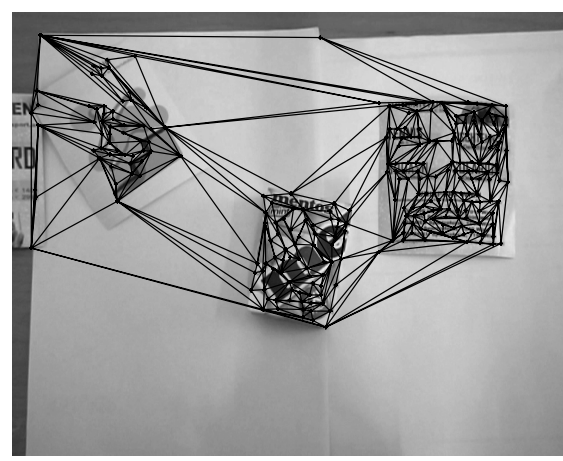

(a) Adaptive triangulation

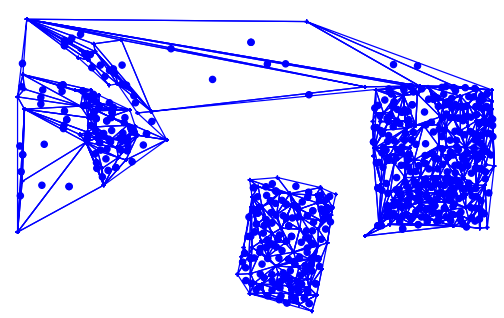

(b) Labeling without "separating"

Fig. 2. Results for video sequence 1. Triangles labeled as separating are not shown. 


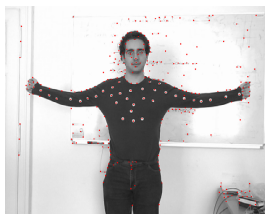

(Frame 1)

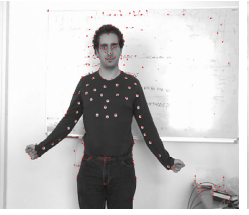

(Frame 422)

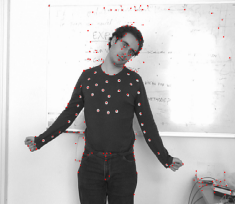

(Frame 455)

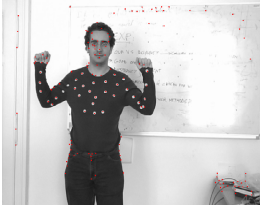

(Frame 663)

Fig. 3. Frames of video sequence 2 that show the movement (articulations) performed

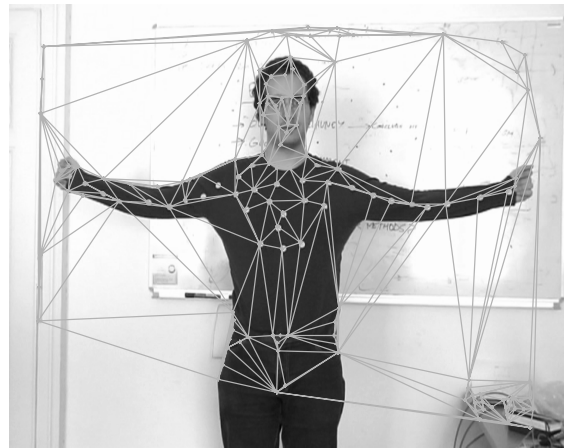

(a) Adaptive triangulation

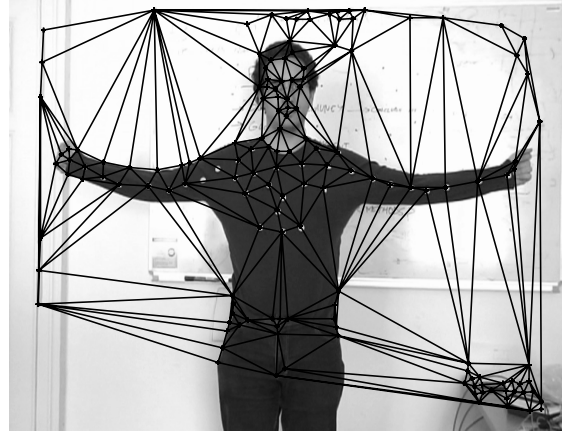

(c) Delaunay triangulation

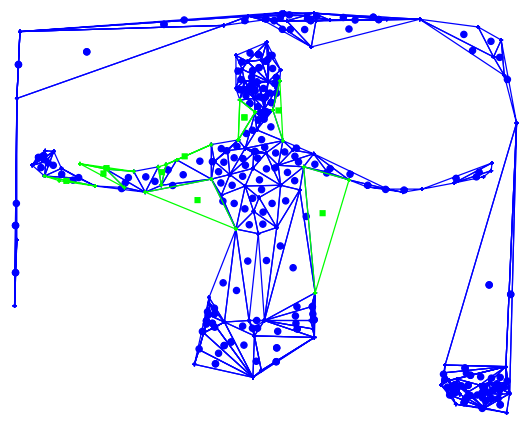

(b) Labeling without "separating"

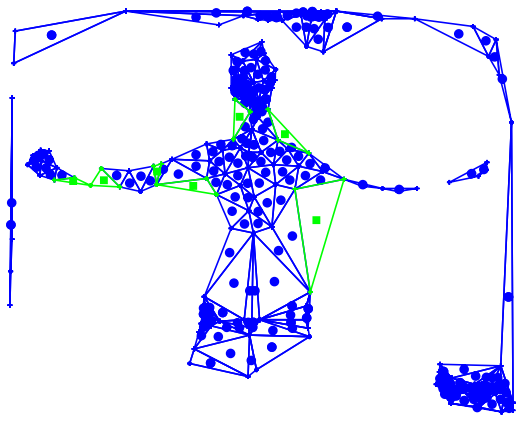

(d) Labeling without "separating"

Fig. 4. Results for video sequence 2. Triangles labeled as separating are not shown.

\section{Conclusion and Future Work}

The adaptive triangulation presented in this paper allows a better description of the structure of a scene than the Delaunay triangulation, because it takes into account the likelihood $l$ of the points belonging together. The spatio-temporal analysis of the movement of the points during the video sequence classifies triangles into rigid, articulated and separating. Our labeling allows to separate foreground and background objects and identifies articulation points. 
In future we plan to combine this approach with kernel-based tracking [9] to track articulated objects. The structural constraints of the graph relaxation can be deduced from the labeled graph. No structural constraints are applied on triangles which are labeled as separating, while for rigid parts the objective is to maintain the tracking consistent with the structure of the object. In the articulated parts edge variations up to a defined degree of freedom are allowed to model articulated motion (e.g. human motion).

\section{References}

1. Celasun, I., Tekalp, A.M., Gokcetekin, M.H., Harmanci, D.M.: 2-d mesh-based video object segmentation and tracking with occlusion resolution. Signal Processing: Image Communication 16(10), 949-962 (2001)

2. Alatan, A.A., Onural, L., Wollborn, M., Mech, R., Tuncel, E., Sikora, T.: Image sequence analysis for emerging interactive multimedia services. IEEE Transactions on Circuits and Systems for Video Technology 8(7), 802-813 (1998)

3. Altunbasak, Y., Eren, P.E., Tekalp, A.M.: Region-based parametric motion segmentation using color information. Graphical Models and Image Processing 60(1), 13-23 (1998)

4. Castagno, R., Ebrahimi, T., Kunt, M.: Video segmentation based on multiple features for interactive multimedia applications. IEEE Transactions on Circuits and Systems for Video Technology 8(5), 562-571 (1998)

5. Chen, H.T., Liu, T.L., Fuh, C.S.: Segmenting highly articulated video objects with weak-prior random forests. In: Leonardis, A., Bischof, H., Pinz, A. (eds.) ECCV 2006. LNCS, vol. 3954, pp. 373-385. Springer, Heidelberg (2006)

6. Celasun, I., Tekalp, A.: Optimal 2-d hierarchical content-based mesh design and update for object-based video. IEEE Transactions on Circuits and Systems for Video Technology 10(7), 1135-1153 (2000)

7. Li, H., Lin, W., Tye, B., Ong, E., Ko, C.: Object segmentation with affine motion similarity measure. In: IEEE International Conference on Multimedia and Expo, 2001, August 22-25. ICME 2001, pp. 841-844 (2001)

8. Tekalp, A., Van Beek, P., Toklu, C., Gunsel, B.: Two-dimensional mesh-based visual-object representation for interactive synthetic/natural digital video. Proceedings of the IEEE 86(6), 1029-1051 (1998)

9. Artner, N., López Mármol, S.B., Beleznai, C., Kropatsch, W.G.: Kernel-based tracking using spatial structure. In: 32nd annual workshop of the Austrian Association for Pattern Recognition, Austria, pp. 103-114 (2008)

10. Matas, J., Chum, O., Urban, M., Pajdla, T.: Robust wide baseline stereo from maximally stable extremal regions. Image and Vision Computing 22(10), 761-767 (2004)

11. Birchfeld, S.: Klt: An implementation of the kanade-lucas-tomasi feature tracker (March 2008), http://www.ces.clemson.edu/ stb/klt/

12. Moss, S., Wilson, R.C., Hancock, E.R.: A mixture model for pose clustering. Pattern Recognition Letters 20(11-13), 1093-1101 (1999)

13. Klette, R., Rosenfeld, A.: Digital Geometry. Morgan Kaufmann, San Francisco (2004)

14. Shi, J., Tomasi, C.: Good features to track. In: Proceedings of the Conference on Computer Vision and Pattern Recognition, pp. 593-600. IEEE Computer Society Press, Los Alamitos (June 1994) 